\title{
Fate of chiral critical point under the strong isospin asymmetry
}

\author{
Hiroaki Abuki* \\ Department of Physics, Tokyo University of Science, Tokyo 162-8601, Japan
}

(Dated: March 11, 2022)

\begin{abstract}
We study the influence of the isospin asymmetry on the phase structure of strongly interacting quark matter near the critical point (CP) using a Ginzburg-Landau approach. The effect is found to be drastic, not only bringing about the shift of the location of the $\mathrm{CP}$, but resulting in a rich phase structure in the vicinity of the CP. In particular, new tricritical and triple points emerge as soon as the isospin density becomes finite. Moreover, we find the CP being washed out from the phase diagram due to the stabilization of a homogeneous charged pion condensate when the isospin chemical potential exceeds a critical value. We derive a model-independent universal relation between the critical isospin chemical potential and the chiral condensate at the CP. We also study the effect of the $\mathrm{U}(1)_{\mathrm{A}}$ anomaly on the phase transition to the pion condensate in the vicinity of chiral crossover.

PACS numbers: 12.38.Mh, 21.65.Qr
\end{abstract}

\section{INTRODUCTION}

The phase diagram of QCD at finite temperature and/or finite density is the subject of extensive theoretical and experimental studies. In particular, several approaches to QCD with two light flavors suggest the existence of a critical point (CP) at which the first-order chiral phase transition turns into a crossover [1]. Despite many efforts based on the first principle calculations [2], not only the precise location of the $\mathrm{CP}$, but its existence itself remains controversial.

In the chiral limit with vanishing quark mass, the $\mathrm{CP}$ becomes a tricritical point (TCP). The effect of a finite quark mass is, thus, rather simple; just to smear the second-order chiral phase transition to a crossover and, accordingly turn the TCP into the CP. Our focus here is the other important ingredient in realistic systems, the effect of an isospin asymmetry. Such a flavor symmetry breaking is caused by a neutrality constraint that should be imposed in any bulk systems to prevent the diverging energy density.

The isospin imbalance is known to bring a rich variety of color superconducting phases at high density [3]. On the other hand, QCD at large isospin density was first studied in Ref. [4], and it was shown that the QCD vacuum develops a pion condensate (PIC) as soon as $\left|\mu_{\mathrm{I}}\right|>m_{\pi}$, where $m_{\pi}$ and $\mu_{\mathrm{I}}$ are the vacuum pion mass and the isospin chemical potential, respectively. The PIC can be viewed as a relativistic superfluid that exhibits a crossover from a Bose-Einstein condensate (BEC) of pions to a superfluidity of the Bardeen-Cooper-Schrieffer (BCS) type [5]. So far, several model analyses have been made for the PIC at finite temperature and/or quark density [5-13]. However, to our knowledge, there is at present, no systematic analysis based on the GinzburgLandau (GL) approach focusing on the isospin effects on the CP. This is what we present here for the first time.

\footnotetext{
* E-mail: h.abuki@rs.tus.ac.jp
}

Our GL framework is advantageous to other approaches in the sense that it can give model-independent predictions near the CP. Since we are interested in the response of the $\mathrm{CP}$ and phases in its neighborhood against nonzero $\mu_{\mathrm{I}}$, our strategy is to take $\mu_{\mathrm{I}}$ as a perturbative field and expand the GL functional with respect to it. We use a quark loop approximation to reduce the number of GL couplings. This approximation should be valid, in particular, if it is located at a large fugacity region.

In this paper, we restrict the analysis to homogeneous phases only, leaving more detailed analysis to future work 14]. This is, to some extent, an extension of our previous work [15] to the situation off the chiral limit introducing a finite quark mass. This paper is organized as follows. In Sec. III. we derive a general GL potential up to the fourth order in fields and discuss the effects of $\mu_{\mathrm{I}}$ and $\mathrm{U}(1)_{\mathrm{A}}$ breaking at the vicinity of chiral crossover. In Sec. III, we extend the GL potential up to the sixth order to discuss the isospin effect on the CP. Based on this, we clarify how the $\mathrm{CP}$ and its neighborhood are affected by the inclusion of isospin asymmetry. In Sec. IV] we summarize.

\section{GINZBURG-LANDAU APPROACH AT FOURTH ORDER}

Let us start with writing the most general GL potential for the two chiral four-vectors $\phi=(\sigma, \boldsymbol{\pi})$ and its parity partner $\varphi=\left(\eta^{\prime}, \boldsymbol{a}\right)$ with $\sigma \sim\langle\bar{q} q\rangle, \boldsymbol{\pi} \sim\left\langle\bar{q} i \gamma^{5} \boldsymbol{\tau} q\right\rangle, \eta^{\prime} \sim$ $\left\langle\bar{q} i \gamma_{5} q\right\rangle$, and $\boldsymbol{a} \sim\langle\bar{q} \boldsymbol{\tau} q\rangle$. At the fourth order in $\phi$ and $\varphi$, the chiral $\left[\mathrm{SU}(2)_{\mathrm{L}} \times \mathrm{SU}(2)_{\mathrm{R}} \sim \mathrm{O}(4)\right]$ symmetric part of the GL potential should take the form

$$
\begin{aligned}
\Omega_{0}[\phi, \varphi]= & \frac{1}{2} \alpha_{2} \phi^{2}+\frac{1}{2} \alpha_{2}^{\prime} \varphi^{2} \\
& +\frac{1}{4} \alpha_{4}\left(\left(\phi^{2}+\varphi^{2}\right)^{2}+4\left(\phi^{2} \varphi^{2}-(\phi, \varphi)^{2}\right)\right),
\end{aligned}
$$

where $(\phi, \varphi) \equiv \sigma \eta^{\prime}+\boldsymbol{a} \cdot \boldsymbol{\pi}$ is the inner product. If $\alpha_{2}=\alpha_{2}^{\prime}$, the potential possesses the additional $\mathrm{U}(1)_{\mathrm{A}}$ symmetry, which is actually violated in QCD via the axial anomaly, so, typically, $\alpha_{2} \neq \alpha_{2}^{\prime}$. The current quark mass and the 
isospin chemical potential add $\mathrm{O}(4)$ noninvariant terms to the potential. At the second order in fields,

$$
\delta \Omega_{\mathrm{SB}}=-h \sigma+\beta_{1}\left(\sigma a_{3}+\eta^{\prime} \pi_{3}\right)+\beta_{2} \boldsymbol{\pi}_{\perp}^{2},
$$

where $\boldsymbol{\pi}_{\perp}=\left(\pi_{1}, \pi_{2}\right)$ is the charged pion doublet. The first term is due to the current quark mass, and it explicitly breaks $\mathrm{O}(4)$ down to $\mathrm{SU}(2)_{\mathrm{V}} \sim \mathrm{O}(3)$. The second and third terms are due to the finite isospin density, which violate the isospin $\mathrm{O}(3)$ into $\mathrm{U}_{\mathrm{I}_{3}}(1) \sim \mathrm{O}(2)$, the rotation about the third axis of isospin space. The GL coupling $h$ is proportional to the quark mass $m$ for light flavors. On the other hand, since the operator in the second (third) term is even (odd) under the isospin flip $u \leftrightarrow d$, we have $\beta_{1} \propto \mu_{\mathrm{I}}$ and $\beta_{2} \propto \mu_{\mathrm{I}}^{2}$ at the leading order in expansion in $\mu_{\mathrm{I}}$. When $\mu_{\mathrm{I}} \neq 0$, and $h \neq 0$, we may anticipate the realization of following two phases:

(i) The chiral symmetry broken phase ( $\chi \mathbf{S B})$ : the phase with $\sigma \neq 0$, which might be accompanied by a nonvanishing $a_{3}$. The residual symmetry is the isospin, $\mathrm{O}(3)$.

(ii) The phase with a pion condensate (PIC): the phase with the charged pion condensate; $\left|\boldsymbol{\pi}_{\perp}\right| \neq 0$. The $\mathrm{O}(3)$ symmetry is spontaneously broken down to $\mathrm{O}(2)$.

We now have six GL couplings $\left\{\alpha_{2}, \alpha_{2}^{\prime}, \alpha_{4}, h, \beta_{1}, \beta_{2}\right\}$, and they are functions of thermodynamic variables $\left\{\mu, \mu_{\mathrm{I}}, T\right\}$. We can, in principle, investigate the phase structure in this six-dimensional space in full generality. However, even if we do that, it would become difficult to relate it with the phase structure in the physical parameter space. Instead of doing that, here we take the advantage of quark loop approximation for which we only take into account quark loop effects in the effective potential. This would give a reasonable approximation to the real potential in the high fugacity region. The feedback of quark loops to the potential is

$$
\Delta \Omega=-\frac{T N_{\mathrm{c}}}{V} \sum_{n=2,4, \cdots} \frac{1}{n} \operatorname{Tr}\left(S_{0} \Sigma\right)^{n},
$$

where $V$ denotes the spatial volume and $\operatorname{Tr}$ should be taken over the Dirac, flavor, and functional indices. $S_{0}=\operatorname{diag}\left(S_{u}, S_{d}\right)$ is the bare quark propagator, and $\Sigma=\sigma \mathbf{1}+a_{3} \tau_{3}+i \gamma^{5} \pi \tau^{1}$ is the self-energy for which we set $\pi_{2}=0$ without any loss of generality. From this we can extract the following explicit expression for $\alpha_{4}$ in the leading order of expansion in $\mu_{\mathrm{I}}$ [16]:

$$
\alpha_{4}=\alpha_{4}^{(0)}(\mu, T)+\mathcal{O}\left(\mu_{\mathrm{I}}^{2}\right)
$$

where we have defined the quantity $\alpha_{2 n}^{(0)}$ for $n \geq 1$ as

$$
\alpha_{2 n}^{(0)}(\mu, T) \equiv 8 T N_{c} \sum_{n, \mathbf{p}} \frac{1}{\left(\left(i \omega_{n}+\mu\right)^{2}-\mathbf{p}^{2}\right)^{n}},
$$

with $\omega_{n}$ being the fermionic Matsubara frequency. Performing the series expansion in $\mu_{\mathrm{I}}$ and discarding parts of integrand containing a total derivative in $\mathbf{p}$ [16], we can relate $\beta_{2}$ and $\beta_{1}$ with $\alpha_{n}^{(0)}$,

$$
\begin{aligned}
& \beta_{1}=\frac{1}{2} \mu_{I} \frac{\partial \alpha_{2}^{(0)}(\mu, T)}{\partial \mu}+\mathcal{O}\left(\mu_{\mathrm{I}}^{3}\right), \\
& \beta_{2}=-\frac{1}{4} \mu_{\mathrm{I}}^{2} \alpha_{4}^{(0)}(\mu, T)+\mathcal{O}\left(\mu_{\mathrm{I}}^{4}\right) .
\end{aligned}
$$

We introduce the GL parameter $\lambda$ by $\left.\left(\beta_{1} / \mu_{\mathrm{I}}\right)\right|_{\mu_{\mathrm{I}}=0}=$ $\lambda \alpha_{4}^{(0)}$; then we have

$$
\lambda=\frac{1}{2 \alpha_{4}^{(0)}} \frac{\partial \alpha_{2}^{(0)}(\mu, T)}{\partial \mu}
$$

which we use instead of $\left.\left(\beta_{1} / \mu_{\mathrm{I}}\right)\right|_{\mu_{\mathrm{I}}=0}$ in the following. In order to find explicit expressions for $\alpha_{2}$ and $\alpha_{2}^{\prime}$ we need to specify the model. Let us consider here for a while the model with four-fermion interaction of the type

$$
\begin{aligned}
\mathcal{L}_{\mathrm{int}}= & \frac{G}{2}\left((\bar{q} q)^{2}+\left(\bar{q} i \gamma_{5} \boldsymbol{\tau} q\right)^{2}+\left(\bar{q} i \gamma_{5} q\right)^{2}+(\bar{q} \boldsymbol{\tau} q)^{2}\right) \\
& +\frac{K}{2}\left((\bar{q} q)^{2}+\left(\bar{q} i \gamma_{5} \boldsymbol{\tau} q\right)^{2}-\left(\bar{q} i \gamma_{5} q\right)^{2}-(\bar{q} \boldsymbol{\tau} q)^{2}\right)
\end{aligned}
$$

The interaction in the second line comes from so-called Kobayashi-Maskawa-'t Hooft determinant, which violates the axial $\mathrm{U}(1)_{\text {A }}$ symmetry explicitly. In this model, we have $\sigma=-(G+K)\langle\bar{q} q\rangle, a_{3}=-(G-K)\left\langle\bar{q} \tau_{3} q\right\rangle$, and

$$
\begin{aligned}
& \alpha_{2}=\frac{1}{G+K}+\alpha_{2}^{(0)}(\mu, T)+\mathcal{O}\left(\mu_{\mathrm{I}}^{6}\right), \\
& \alpha_{2}^{\prime}=\frac{1}{G-K}+\alpha_{2}^{(0)}(\mu, T)+\mathcal{O}\left(\mu_{\mathrm{I}}^{6}\right) .
\end{aligned}
$$

We consider the following two cases here:

Case (I): Strong U(1) A breaking with $K=G$. This corresponds to the standard $\mathrm{Nambu}-$ Jona-Lasinio (NJL) model with

$$
\mathcal{L}_{\mathrm{int}}=G\left((\bar{q} q)^{2}+\left(\bar{q} i \gamma_{5} \boldsymbol{\tau} q\right)^{2}\right)
$$

In this case, $\alpha_{2}^{\prime}$ diverges so that one of the chiral fourvectors $\varphi$ becomes irrelevant. In particular, $a_{3}=0$, 17.

Case (II): U(1) A symmetric case with $K=0$. In this case, $\alpha_{2}=\alpha_{2}^{\prime}$ so that we see that the GL potential also possesses the symmetry as it should.

For case I, we find up to the fourth order in fields and $\mu_{\mathrm{I}}$

$$
\Omega=\frac{\alpha_{2}}{2} \phi^{2}+\frac{\alpha_{4}^{(0)}}{4} \phi^{4}-h \sigma-\frac{\alpha_{4}^{(0)}}{4} \mu_{\mathrm{I}}^{2} \pi_{\perp}^{2} .
$$

Here, $\alpha_{2}$ should be regarded as one evaluated at $\mu_{\mathrm{I}}=$ 0 , i.e., it should be understood as the lowest order in expansion in $\mu_{\mathrm{I}}$, Eq. (77). If we introduce the notation

$$
\sigma_{u}=\frac{\sigma+a_{3}}{2}, \quad \sigma_{d}=\frac{\sigma-a_{3}}{2}
$$

then we are forced to have $\sigma_{u}=\sigma_{d}=\sigma / 2$. 


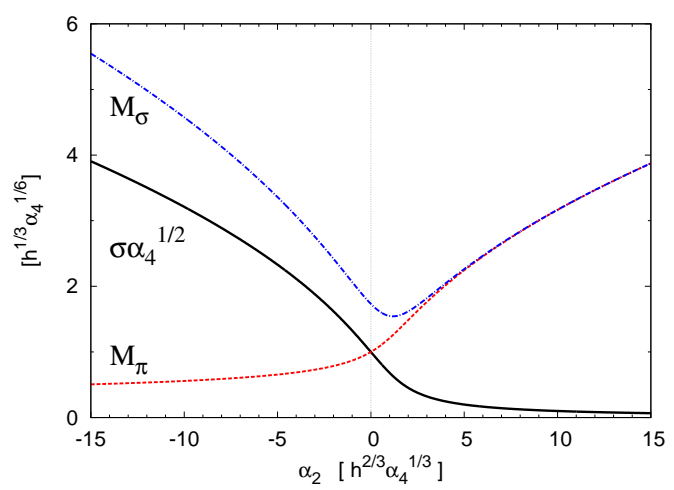

FIG. 1. The chiral condensate $\sigma$ (solid), the sigma mass $M_{\sigma}$ (dashed, blue), and the pion mass $M_{\pi}$ (dotted, red) as a function of $\alpha_{2}$, at $\mu_{\mathrm{I}}=0$.

For case II, we have at the same order

$$
\begin{aligned}
\Omega= & \frac{\alpha_{2}}{2}\left(\phi^{2}+\varphi^{2}\right) \\
& +\frac{\alpha_{4}^{(0)}}{4}\left(\left(\phi^{2}+\varphi^{2}\right)^{2}+4\left(\phi^{2} \varphi^{2}-(\phi, \varphi)^{2}\right)\right) \\
& -h \sigma+\frac{\alpha_{4}^{(0)}}{4} \mu_{\mathrm{I}}^{2} \pi_{\perp}^{2}+\alpha_{4}^{(0)} \lambda \mu_{\mathrm{I}}\left(\sigma a_{3}+\eta^{\prime} \pi_{3}\right) .
\end{aligned}
$$

For stability, we can only investigate the region $\alpha_{4}>0$ at this order, and we have typically $\lambda \geq 0$ for $\mu \geq 0$. $\sigma_{u}$ and $\sigma_{d}$ in this case are proportional to $\bar{u} u$ and $\bar{d} d$ condensates. We notice that the $\lambda$ term in the potential contains $\lambda \alpha_{4} \mu_{\mathrm{I}}\left(\sigma_{u}^{2}-\sigma_{d}^{2}\right)$ so that positive $\mu_{\mathrm{I}}$ favors the situation $\sigma_{d}>\sigma_{u}$. In the following analysis, we suppress the subscript (0) in couplings, so we have $\mu_{\mathrm{I}}$ and four independent parameters $\left\{\alpha_{2}, \alpha_{4}, h, \lambda\right\}$, which do not depend on $\mu_{\mathrm{I}}$.

Let us first investigate case I. Introducing dimensionless parameters $\tilde{\sigma}, \tilde{\boldsymbol{\pi}}, \tilde{\mu_{\mathrm{I}}}$, and $\tilde{\alpha_{2}}$ via

$$
\begin{aligned}
& \sigma=\tilde{\sigma}\left(h / \alpha_{4}\right)^{1 / 3}, \quad \boldsymbol{\pi}=\tilde{\boldsymbol{\pi}}\left(h / \alpha_{4}\right)^{1 / 3}, \\
& \alpha_{2} \equiv \tilde{\alpha}_{2} h^{2 / 3} \alpha_{4}^{1 / 3}, \quad \mu_{\mathrm{I}} \equiv \tilde{\mu}_{\mathrm{I}}\left(h / \alpha_{4}\right)^{1 / 3},
\end{aligned}
$$

the potential is cast into $\Omega=\alpha_{4}^{-1 / 3} h^{4 / 3} \omega$, where $\omega$ does not have any explicit dependence on $h$ :

$$
\omega\left(\tilde{\sigma}, \tilde{\boldsymbol{\pi}} ; \tilde{\alpha}_{2}, \tilde{\mu_{\mathrm{I}}}\right) \equiv \frac{\tilde{\alpha}_{2}}{2} \tilde{\phi}^{2}+\frac{1}{4} \tilde{\phi}^{4}-\tilde{\sigma}-\frac{1}{4} \tilde{\mu}_{\mathrm{I}}^{2} \tilde{\boldsymbol{\pi}}_{\perp}^{2},
$$

with $\tilde{\phi} \equiv(\tilde{\sigma}, \tilde{\boldsymbol{\pi}})$. When $h=0$ and $\mu_{\mathrm{I}}=0$, the system has a second-order phase transition at $\alpha_{2}=0$. At finite $h \neq 0$, the transition gets smoothed to a crossover. In Fig. 1, we show the behavior of chiral condensate $\sigma$, sigma and pion masses $\left(M_{\sigma}, M_{\pi}\right)$ as a function of $\alpha_{2}$. We find a crossover from the $\chi \mathrm{SB}$ to an approximately restored phase with $\sigma \sim 0$ when $\alpha_{2}$ is increased. We can define the pseudocritical point $\alpha_{2}=\alpha_{2}^{\mathrm{pc}}$, for example, by the point where $M_{\sigma}$ takes the minimum. In this case, it can be numerically read as

$$
\alpha_{2}^{\mathrm{pc}}=1.191 h^{2 / 3} \alpha_{4}^{1 / 3} .
$$

At this point, pion and sigma masses are

$$
M_{\sigma}^{\mathrm{pc}}=1.54 h^{1 / 3} \alpha_{4}^{1 / 6}, \quad M_{\pi}^{\mathrm{pc}}=1.26 h^{1 / 3} \alpha_{4}^{1 / 6} .
$$

The ratio $M_{\sigma}^{\mathrm{pc}} / M_{\pi}^{\mathrm{pc}}=1.22$ at the pseudocritical point is universal to this order of GL expansion. The chiral condensate $\sigma$ is also read as

$$
\sigma=0.630 h^{1 / 3} \alpha_{4}^{-1 / 3}\left(\equiv \sigma_{\mathrm{pc}}\right) .
$$

For what follows, we concentrate on the effect of $\mu_{\mathrm{I}}$ at the pseudocritical point. In Fig. 2, we show $\sigma_{u}, \sigma_{d}$ and $\left|\boldsymbol{\pi}_{\perp}\right| \equiv \pi$ as a function of $\mu_{\mathrm{I}}$. We see a second-order phase transition to the PIC phase at

$$
\left|\mu_{\mathrm{I}}\right|=1.782 h^{1 / 3} \alpha_{4}^{-1 / 3}\left(\equiv \mu_{\mathrm{I}}^{\mathrm{c}}\right) .
$$

This can be written in terms of $\sigma_{\mathrm{pc}}$ or $M_{\pi}^{\mathrm{pc}}$ as

$$
\mu_{\mathrm{I}}^{\mathrm{c}}=2.83 \sigma_{\mathrm{pc}}=\left(1.41 / \sqrt{\alpha_{4}}\right) M_{\pi}^{\mathrm{pc}} .
$$

We notice that what is in the universal relation in the GL framework at this order is the ratio of $\mu_{\text {I }}$ to the flavor singlet quark condensate $\sigma$ rather than that to the pion mass $M_{\pi}$. Once $\left|\mu_{\mathrm{I}}\right| \geq \mu_{\mathrm{I}}^{\mathrm{c}}$, the charged pion condensate $\pi$ develops.

A couple of questions are in order here: i) First, one might think that $\mu_{\mathrm{I}}^{\mathrm{c}}$ determined here by looking at the static correlation function might be different from the true one, which should be determined by the pole of the charged pion propagator due to the kinetic seesaw mechanism [18, 19]. ii) Second, one might wonder what is the difference of $\pi^{+}$and $\pi^{-}$condensations. Let us first discuss the point i. Actually, this is not the case, and the critical point determined by the static effective potential exactly coincides with the one by the charged pion propagators. If we worked out the time-derivative expansion in charged pion fields within the Gaussian approximation, we would have obtained the effective action density, which looks like (with a suitable normalization of $\pi$ fields)

$$
\begin{aligned}
\mathcal{L}_{\text {eff }}= & \operatorname{tr}\left[\left(i \partial_{t} \boldsymbol{\pi}_{\perp}+\left[\hat{\mu}_{\mathrm{I}}, \boldsymbol{\pi}_{\perp}\right]\right)^{\dagger}\left(i \partial_{t} \boldsymbol{\pi}_{\perp}+\left[\hat{\mu}_{\mathrm{I}}, \boldsymbol{\pi}_{\perp}\right]\right)\right] \\
& -\frac{1}{2} M^{2}\left(\pi_{1}^{2}+\pi_{2}^{2}\right),
\end{aligned}
$$

where $M$ is the mass parameter being a function of microscopic/thermodynamic variables, $\boldsymbol{\pi}_{\perp} \equiv\left(\pi_{1} \tau_{1}+\pi_{2} \tau_{2}\right) / 2$ and $\hat{\mu}_{\mathrm{I}} \equiv \mu_{\mathrm{I}} \tau_{3} / 2$, with $\left\{\tau_{1}, \tau_{2}, \tau_{3}\right\}$ being the Pauli matrices. The charged pion propagator can be read as

$$
D_{\pi_{i} \pi_{j}}^{-1}(\omega)=\left(\begin{array}{cc}
-\omega^{2}+M^{2}-\mu_{\mathrm{I}}^{2} & -2 i \mu_{\mathrm{I}} \omega \\
+2 i \mu_{\mathrm{I}} \omega & -\omega^{2}+M^{2}-\mu_{\mathrm{I}}^{2}
\end{array}\right) .
$$

The static part of the propagator is related with the second derivative of effective potential:

$$
M^{2}-\left.\mu_{\mathrm{I}}^{2} \equiv \frac{\partial^{2} \Omega}{\partial \pi_{1}^{2}}\right|_{\boldsymbol{\pi}_{\perp}=0}=\left.\frac{\partial^{2} \Omega}{\partial \pi_{2}^{2}}\right|_{\boldsymbol{\pi}_{\perp}=0} .
$$

The determinant of the polarization matrix includes all the pole masses:

$$
\operatorname{det} D_{\pi_{i} \pi_{j}}^{-1}=\left(\omega^{2}-M_{+}^{2}\right)\left(\omega^{2}-M_{-}^{2}\right)
$$




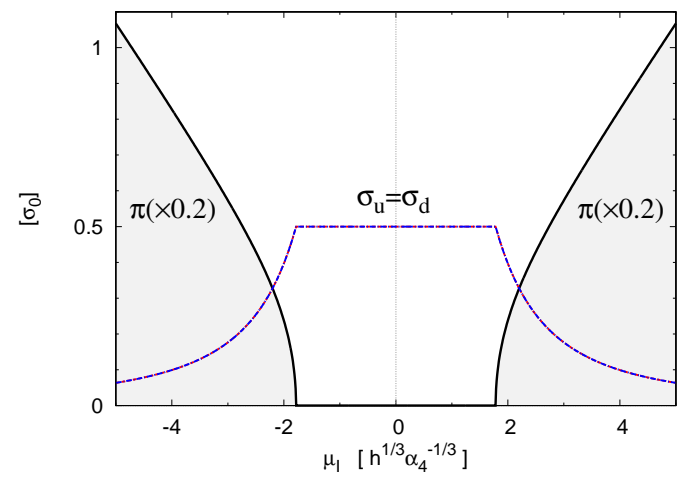

FIG. 2. The behavior of condensates as a function of $\mu_{\mathrm{I}}$ at the crossover point, Eq. (12). $\sigma_{u}$ and $\sigma_{d}\left(=\sigma_{u}\right)$ are depicted by dashed lines (red and blue, respectively), while $\pi$ is shown by a solid line.

$M_{+} \equiv M-\mu_{\text {I }}$ corresponds to the $\pi^{+}$pole, while $M_{-} \equiv$ $M+\mu_{\mathrm{I}}$ represents the $\pi^{-}$pole. The critical condition is given by the vanishing of either $\pi^{+}$or $\pi^{-}$mass, and, in both cases, $M^{2}-\mu_{\mathrm{I}}^{2}=M_{+} M_{-}=\sqrt{\left.\operatorname{det} D_{\pi_{i} \pi_{j}}^{-1}\right|_{\omega \rightarrow 0}}=0$. This is equivalent with the condition

$$
\left.\frac{\partial^{2} \Omega}{\partial \pi_{1}^{2}}\right|_{\pi_{\perp}=0}=\left.\frac{\partial^{2} \Omega}{\partial \pi_{2}^{2}}\right|_{\pi_{\perp}=0}=0
$$

This means that even if the pion masses split due to the kinetic seesaw mechanism, the critical chemical point can be always obtained by looking at the behavior of static susceptibility. Of course, we can only extract from $\Omega$ the multiple of two poles $M_{+} M_{-}$, not separately $M_{+}$ and $M_{-}$. In other words, it is the geometric average of the pole masses that can be read from the curvature mass of $\Omega$. Let us now come to point ii. Since the time dependence of the charged condensate is determined by the chemical potential as $\pi^{+}=\pi_{1}-i \pi_{2} \sim e^{-i \mu_{1} t} \pi$, i.e., $\left(\pi_{1}, \pi_{2}\right) \sim\left(\pi \cos \mu_{\mathrm{I}} t, \pi \sin \mu_{\mathrm{I}} t\right)$, the rotation is counterclockwise when $\mu_{\mathrm{I}}>0$ while it is clockwise for $\mu_{\mathrm{I}}<0$. We shall refer to the former as $\pi^{+}$condensation, and the latter as $\pi^{-}$condensation.

Now, let us move on to case II. In this case, we have an additional GL parameter $\lambda \geq 0$ for $\mu \geq 0$. In the upper panel of Fig. 3, we display the phase diagram in the twodimensional GL parameter space: the $\left(\mu_{\mathrm{I}}, \lambda\right)$ plane. We have basically two phases, the $\chi \mathrm{SB}$ and the PIC with a $\pi^{+}$or $\pi^{-}$condensate. However, in this case, we have $\sigma_{u}=\sigma_{d}$ only on two lines specified by $\mu_{\mathrm{I}}=0$ or $\lambda=0$ and $\sigma_{u} \neq \sigma_{d}$ in the major part of the plane. This is, of course, because of the $\lambda$ term in the GL potential, $\alpha_{4} \lambda \mu_{\mathrm{I}}\left(\sigma_{u}^{2}-\sigma_{d}^{2}\right)$. On the line $\lambda=0$, we have completely the same situation as displayed in Fig. 2 in which two second-order phase transitions are found at $\mu_{\mathrm{I}}= \pm \mu_{\mathrm{I}}^{\mathrm{c}}$. On the other hand, when $\lambda$ becomes large, the transitions eventually change to the first-order ones. We show the situation at $\lambda=1\left[h^{1 / 3} \alpha_{4}^{-1 / 3}\right]$ in the lower panel of Fig. [3,
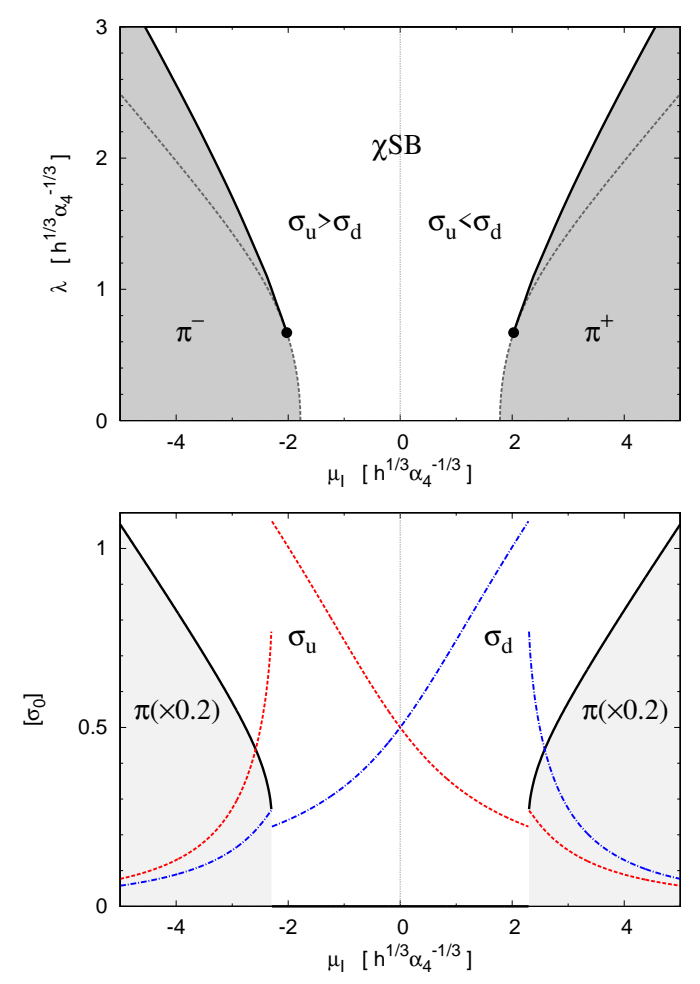

FIG. 3. (Upper panel): The GL phase diagram in the $\left(\mu_{\mathrm{I}}, \lambda\right)$ plane. The solid line represents the first-order phase transition, while the dashed line represents second-order one separating the $\chi \mathrm{SB}$ and PIC phases. The dashed line inside the shaded region expresses the spinodal line where the state without pion condensate becomes unstable. (Lower panel): The behavior of $\sigma_{u}$ (dashed, red), $\sigma_{d}$ (dotted-dashed, blue), and $\pi$ (solid) as a function of $\mu_{\mathrm{I}}$ at $\lambda=1 h^{1 / 3} \alpha_{4}^{-1 / 2}$.

We see clearly finite abrupt gaps in the order parameters associated with the first-order phase transition. This is attributed to the competition between the $\lambda$ term and the $\mu_{\mathrm{I}}^{2}$ term in the potential; the former favors a larger $\mid \sigma_{u}-$ $\sigma_{d} \mid$, while the latter likes the situation $\pi \neq 0$ in which a smaller $\left|\sigma_{u}-\sigma_{d}\right|$ is favorable. Accordingly, there are two tricritical points $\left(\mu_{\mathrm{I}}, \lambda\right)=\left( \pm \mu_{\mathrm{I}}^{\mathrm{TCP}}, \lambda^{\mathrm{TCP}}\right)$ at which the second-order phase transitions turn into first order ones. Numerically, we find

$$
\begin{aligned}
\mu_{\mathrm{I}}^{\mathrm{TCP}} & =2.02 h^{1 / 3} \alpha_{4}^{-1 / 3}=3.21 \sigma_{\mathrm{pc}} \\
\lambda^{\mathrm{TCP}} & =0.67 h^{1 / 3} \alpha_{4}^{-1 / 3}=1.06 \sigma_{\mathrm{pc}} .
\end{aligned}
$$

The ratio $\lambda^{\mathrm{TCP}} / \mu_{\mathrm{I}}^{\mathrm{TCP}}=0.33$ does not depend on any of GL parameters and, thus, is universal to this order. We note that the effect of strong flavor mixing due to the $\mathrm{U}(1)_{\mathrm{A}}$ anomaly makes the $\lambda$ term irrelevant via locking the condensates $\sigma_{u}$ and $\sigma_{d}$ with the same value [9, 17], and, thus, renders the transition a second-order one. 


\section{THE GINZBURG-LANDAU APPROACH AT SIXTH ORDER}

We now extend the GL analysis up to the sixth order so as to explore the influence of $\mu_{\mathrm{I}}$ near the CP of QCD. Doing this in full generality introduces many new GL parameters, which makes the analysis quite complicated. Instead of doing this, we examine here only case I in the previous section, in which one of chiral four-vectors, $\varphi$, decouples. The general GL for homogeneous condensates can be again written in terms of $\phi[15]$

$$
\begin{aligned}
\Omega= & -h \sigma+\frac{\alpha_{2}}{2} \phi^{2}-\beta_{2} \pi_{\perp}^{2} \\
& +\frac{\alpha_{4}}{4} \phi^{4}+\frac{\beta_{4}}{4} \pi_{\perp}^{4}+\frac{\beta_{4 b}}{4}\left(\phi^{2}-\pi_{\perp}^{2}\right) \pi_{\perp}^{2}+\frac{\alpha_{6}}{6} \phi^{6} .
\end{aligned}
$$

GL coefficients $\alpha_{n}(n=2,4,6)$ are expanded in the series of $\mu_{\mathrm{I}}$ within the quark loop approximation (up to total derivatives) as

$$
\left(\begin{array}{l}
\alpha_{2} \\
\alpha_{4} \\
\alpha_{6}
\end{array}\right)=\left(\begin{array}{ccc}
1 & a \mu_{\mathrm{I}}^{2} & b \mu_{\mathrm{I}}^{4} \\
0 & 1 & c \mu_{\mathrm{I}}^{2} \\
0 & 0 & 1
\end{array}\right)\left(\begin{array}{l}
\alpha_{2}\left(\mu_{\mathrm{I}}=0\right) \\
\alpha_{4}\left(\mu_{\mathrm{I}}=0\right) \\
\alpha_{6}\left(\mu_{\mathrm{I}}=0\right)
\end{array}\right) .
$$

Via explicit computations, we find $a=b=0$, and $c=1$. Similarly, for $\beta_{2}, \beta_{4}$ 's we find

$$
\left(\begin{array}{c}
\beta_{2} \\
\left\{\beta_{4}, \beta_{4 b}\right\}
\end{array}\right)=\mu_{\mathrm{I}}^{2}\left(\begin{array}{cc}
d & e \mu_{\mathrm{I}}^{2} \\
0 & \left\{f, f_{b}\right\}
\end{array}\right)\left(\begin{array}{c}
\alpha_{4}\left(\mu_{\mathrm{I}}=0\right) \\
\alpha_{6}\left(\mu_{\mathrm{I}}=0\right)
\end{array}\right),
$$

with $d=-1 / 4, e=0$, and $f=f_{b}=-2$. Putting them all together, and assuming the condensate to have a charged pion component, i.e., $\phi=(\sigma, \pi, 0,0)$, we arrive at

$$
\begin{aligned}
\Omega= & -h \sigma+\frac{\alpha_{2}}{2} \sigma^{2}+\frac{\alpha_{2}-\mu_{\mathrm{I}}^{2} \alpha_{4} / 2}{2} \pi^{2} \\
& +\frac{\alpha_{4}+\mu_{\mathrm{I}}^{2} \alpha_{6}}{4} \sigma^{4}+\frac{\alpha_{4}+\mu_{\mathrm{I}}^{2} \alpha_{6} / 2}{2} \sigma^{2} \pi^{2}+\frac{\alpha_{4}}{4} \pi^{4} \\
& +\frac{\alpha_{6}}{6}\left(\sigma^{2}+\pi^{2}\right)^{3} .
\end{aligned}
$$

Here $\alpha_{2}, \alpha_{4}$ and $\alpha_{6}$ should be understood as those evaluated at $\mu_{\mathrm{I}}=0$ and, thus, are functions of $\mu$ and $T$ only. $\alpha_{6}^{-1 / 2}$ has a dimension of energy so we use this as the unit of energy. In the following analysis, we, thus, set $\alpha_{6}=1$, but the proper dimension of any quantity can be recovered any time by use of $\alpha_{6}^{-1 / 2}$. Now, assuming $h>0$, we get rid of $h$ via scaling

$$
\begin{aligned}
\sigma & =\tilde{\sigma} h^{1 / 5}, \quad \pi=\tilde{\pi} h^{1 / 5}, \quad \mu_{\mathrm{I}}=\tilde{\mu}_{\mathrm{I}} h^{1 / 5}, \\
\alpha_{2} & =\tilde{\alpha}_{2} h^{4 / 5}, \quad \alpha_{4}=\tilde{\alpha}_{4} h^{2 / 5} .
\end{aligned}
$$

Then, $h$ is scaled out of the potential as $\Omega=h^{6 / 5} \omega$. Therefore, we now need to explore the GL phase diagram in the three-dimensional GL parameter space $\left(\alpha_{2}, \alpha_{4}, \mu_{\mathrm{I}}\right)$.

How does $\left(\alpha_{2}, \alpha_{4}\right)$ map onto the $(\mu, T)$-plane? Before going into the discussion of GL phase diagrams, let us briefly sketch how the GL parameters $\left(\alpha_{2}, \alpha_{4}\right)$ map onto the $(\mu, T)$ plane taking the chiral limit $(h=0)$ for simplicity. Figure 4 illustrates how $\left(\alpha_{2}, \alpha_{4}\right)$ spans the local

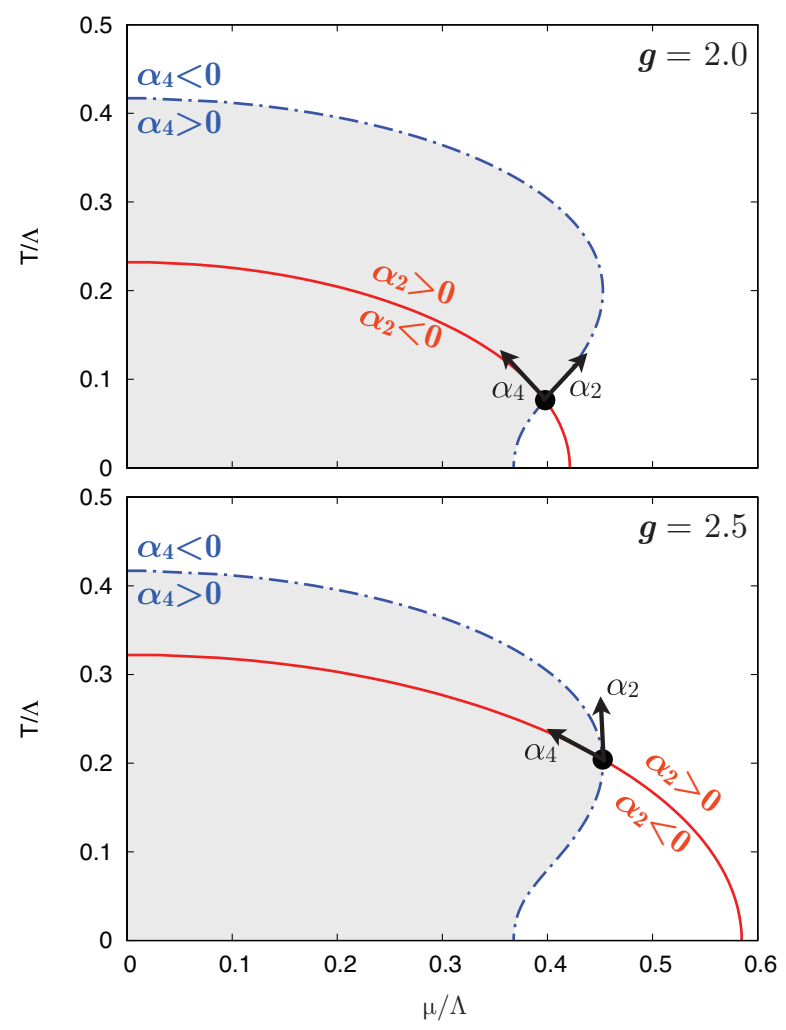

FIG. 4. The illustrative figure that shows how $\left(\alpha_{2}, \alpha_{4}\right)$ spans the local coordinate in the vicinity of the TCP within the NJL model defined by Eq. (8) and the three-momentum cutoff $\Lambda$. This is depicted for two values of coupling $g \equiv G \Lambda^{2}=2.0$ (upper panel) and $g=2.0$ (lower panel). The solid line (red online) shows the curve on which $\alpha_{2}=0$, while the dotteddashed line (blue online) does that for $\alpha_{4}=0$. The point of intersection gives the location of the TCP. The region for $\alpha_{4}>0$ is shaded. The solid line in the shaded area represents the second-order chiral transition, while that in the unshaded area only gives the spinodal line on which the Wigner phase with $\sigma=0$ ceases to be a local minimum.

coordinate in the NJL model specified by Eq. (8) and three-momentum cutoff $\Lambda$. The upper panel shows the situation for $g=G \Lambda^{2}=2.5$, while the lower panel shows that for $g=2.0$. In the figure, the solid line expresses the curve determined by $\alpha_{2}=0$, which separates the $(\mu, T)$ plane into two regions, one for $\alpha_{2}>0$ and the other for $\alpha_{2}<0$. Similarly, the dotted-dashed line shows the curve for $\alpha_{4}=0$. The point of intersection determines the location of the TCP. The region $\alpha_{4}>0$ is shaded just for a guide. The solid line inside this region determines the second-order chiral phase transition, while that outside it only specifies the spinodal line on which the Wigner phase ceases to be even a local minimum. The axises of the local coordinate system $\left(\alpha_{2}, \alpha_{4}\right)$ are depicted by arrows starting from the TCP; the $\alpha_{2}\left(\alpha_{4}\right)$ coordinate points to the direction for $\alpha_{2}>0\left(\alpha_{4}>0\right)$ side with being tangent to the line of $\alpha_{4}=0\left(\alpha_{2}=0\right)$. The location of the TCP as well as how $\left(\alpha_{2}, \alpha_{4}\right)$ maps onto the 


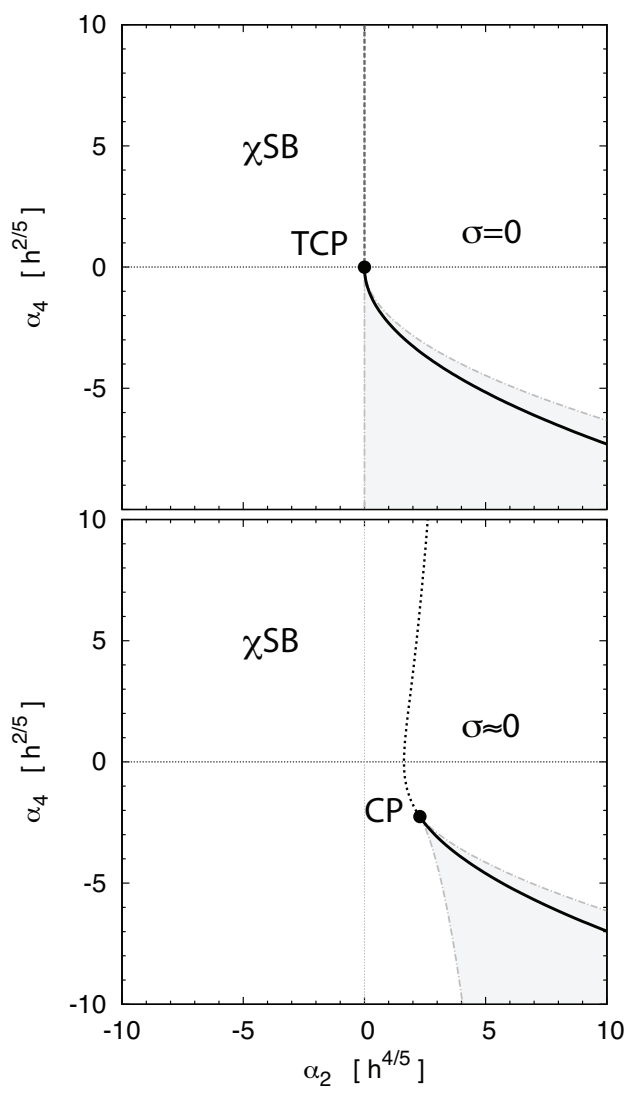

FIG. 5. The GL phase diagrams in the absence of $\mu_{\mathrm{I}}$. The phase diagram in the chiral limit $h=0$ (upper panel) and the one off the chiral limit (lower panel). The shaded area represents the spinodal region. See the text for detail.

$(\mu, T)$ plane depends on the detail of the model or regularization scheme, so, for what follows, we only discuss the phase diagram in the $\left(\alpha_{2}, \alpha_{4}\right)$ plane.

$G L$ phase diagram for quark matter without an isospin imbalance: Let us start with the case with $\mu_{\mathrm{I}}=h=0$. In the upper panel of Fig. 囵, the phase diagram for this case is displayed. In this case, $h$ in the figure labels can be regarded as an arbitrary unit; changing it does not modify the phase diagram. There are two phases: the $\chi \mathrm{SB}$ phase with $\sigma \neq 0$ and the symmetric (Wigner) phase. For $\alpha_{4}>0$, these phases are separated by a second-order phase transition located at $\alpha_{2}=0$, which is depicted by the dashed line. For $\alpha_{4}<0$, the transition is replaced by the first-order one at $\alpha_{2}=\frac{3}{16} \alpha_{4}^{2}$ shown by a solid line [16]. Accordingly, the TCP is located at the origin. The shaded area shows a spinodal region in which one of the states exists as a local minimum of the potential; at $\alpha_{2}=0$ for $\alpha_{4}<0$, the Wigner phase becomes unstable, while at the line $\alpha_{2}=\frac{1}{4} \alpha_{4}^{2}$ for $\alpha_{4}<0$, a local minimum corresponding to the $\chi \mathrm{SB}$ state vanishes. In the lower panel of Fig. 5 we show the phase diagram for $h \neq 0$. In this case, we have only the situation $\sigma \neq 0$. Nevertheless, the first-order phase transition survives and separates the $\chi \mathrm{SB}$ phase and a nearly symmetric phase with $\sigma \sim 0$. The first-order phase transition ends at the $\mathrm{CP}$. The exact location of the $\mathrm{CP},\left(\alpha_{2}^{\mathrm{CP}}, \alpha_{4}^{\mathrm{CP}}\right)$, is derived analytically [20]

$$
\left(\alpha_{2}^{\mathrm{CP}}, \alpha_{4}^{\mathrm{CP}}\right)=\left(\frac{5}{4} \frac{3^{4 / 5}}{2^{2 / 5}} h^{4 / 5},-\frac{5}{2^{1 / 5} 3^{3 / 5}} h^{2 / 5}\right) .
$$

This is numerically evaluated as $\sim\left(2.28 h^{4 / 5},-2.25 h^{2 / 5}\right)$. The chiral condensate at this point is found as

$$
\sigma=0.822 h^{1 / 5}\left(\equiv \sigma_{0}\right) .
$$

The dotted line starting from the CP expresses the pseudocritical line determined by the location of the minimum in the sigma meson mass. The shaded area again represents the spinodal region, in which there is another state competing with the ground state.

GL phase diagram for quark matter with an isospin imbalance: Now, we discuss the influence of $\mu_{\mathrm{I}}$ on the phase diagram. The phase diagrams for several finite values of $\mu_{\mathrm{I}}$ are shown in Fig. 6. From Fig.6(a) to Fig. 6(d), the value of $\mu_{\mathrm{I}}$ increases. We now suppress the spinodal region surrounding the line of the first-order chiral phase transition.

Let us start with (a), where the value of $\mu_{\mathrm{I}}$ is set to $\mu_{\mathrm{I}}^{2}=0.2 h^{2 / 5}$, that is, in terms of $\sigma_{0}, \mu_{\mathrm{I}}=0.54 \sigma_{0}$. In this case we notice that the structure in the proximity of the $\mathrm{CP}$ is unaffected even though the location of the $\mathrm{CP}$ is shifted downward according to

$$
\left(\alpha_{2}^{\mathrm{CP}}, \alpha_{4}^{\mathrm{CP}}\right) \rightarrow\left(\alpha_{2}^{\mathrm{CP}}, \alpha_{4}^{\mathrm{CP}}-\mu_{\mathrm{I}}^{2}\right)
$$

This can be easily understood from the coefficient of the $\sigma^{4}$ term in Eq. (26). Recalling the direction in which the local coordinate $\alpha_{4}$ points in the $(\mu, T)$ plane (Fig. (4), we expect that the $\mathrm{CP}$ shifts to the direction of lower temperature and higher chemical potential due to the isospin asymmetry. This is consistent with the analysis done in Ref. 21]. Moreover, a recent analysis within a specific model shows at some critical value of $\mu_{\text {I }}$ the TCP (CP) can even touch the $\mu$ axis disappearing eventually from the phase diagram [22]. Apart from this simple shift of chiral phase transition, we notice that the continent of the PIC dominates the region deep in the $\chi \mathrm{SB}$. The PIC and $\chi \mathrm{SB}$ phases are separated by a second-order phase transition in all regions shown in the figure.

Figure 6(b) shows how the situation changes when $\mu_{\mathrm{I}}$ is increased to $\mu_{\mathrm{I}}^{2}=0.5 h^{2 / 5}$, that is, $\mu_{\mathrm{I}} \cong 0.86 \sigma_{0}$. We notice that the $\mathrm{CP}$ moves further downward according to Eq. (30), and the continent of the PIC gets wider as expected. Moreover, the transition from the $\chi \mathrm{SB}$ to the PIC now has a branch of the first-order phase transition, which is drawn by a solid line. Accordingly, there appears a new tricritical point denoted by $\mathrm{TCP}^{\prime}$ on the critical line. This is actually the tricritical point at which three critical lines meet up, once we introduce an external field for the charged pion condensate. The line for the first-order phase transition departing from the $\mathrm{TCP}^{\prime}$ encounters the line of the first-order chiral phase transition 


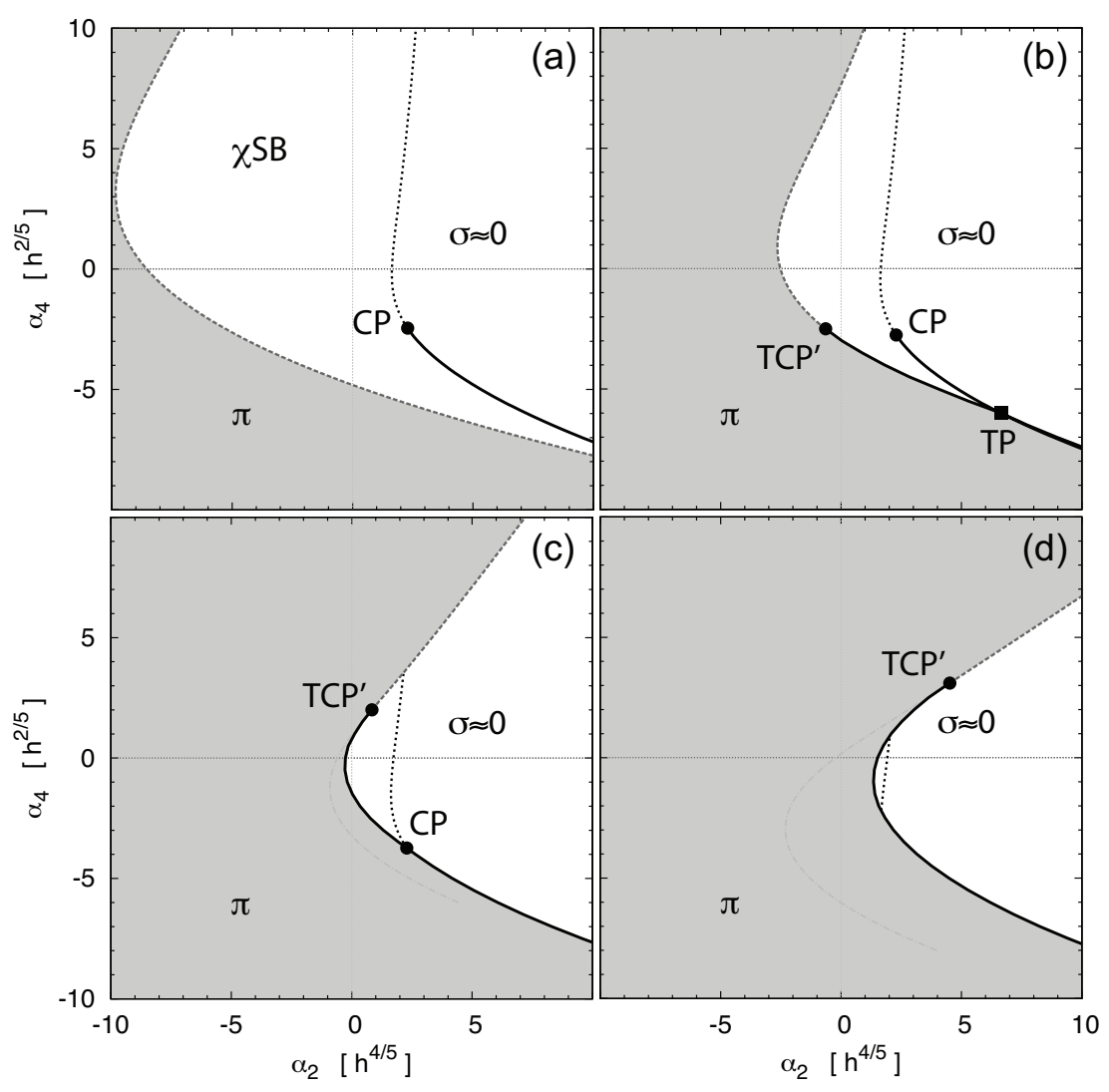

FIG. 6. The GL phase diagrams for several values of $\mu_{\mathrm{I}}$; from (a) to (d), $\mu_{\mathrm{I}}$ increases as (a) $\mu_{\mathrm{I}}^{2}=0.2 h^{2 / 5},(\mathrm{~b}) \mu_{\mathrm{I}}^{2}=0.5 h^{2 / 5}$, (c) $\mu_{\mathrm{I}}^{2} \equiv\left(\mu_{\mathrm{I}}^{\mathrm{c}}\right)^{2}$, and (d) $\mu_{\mathrm{I}}^{2}=3.0 h^{2 / 5}$. For $\mu_{\mathrm{I}}^{\mathrm{c}}$, see the text.

at the point "TP", which stands for the triple point. At the triple point, three phases - the $\chi \mathrm{SB}$, a nearly restored phase, and the PIC - coexist and compete.

Now, let us discuss Fig. 6(d) before Fig. 6(c). In Fig. 6(d), $\mu_{\mathrm{I}}$ is set to a large value $\mu_{\mathrm{I}}^{2}=3.0 h^{2 / 5}$, which corresponds to $\mu_{\mathrm{I}} \cong 2.1 \sigma_{0}$. In this case, the PIC dominates a major part of the phase diagram, and the structure of the chiral phase transition is now completely hidden. There remains a dotted line outside the PIC, which is just the chiral crossover. The transition from the PIC to the $\chi \mathrm{SB}$ phase becomes widely of first order, and, accordingly, the location of the $\mathrm{TCP}^{\prime}$ is shifted upward.

Since the CP is completely hidden in Fig. 6(d), there should be a critical value of $\mu_{\mathrm{I}}$ at which the $\mathrm{CP}$ vanishes from the phase diagram. In Fig. 6(c), we show this situation. The critical chemical potential is $\mu_{\mathrm{I}}^{2}=1.477 \mathrm{~h}^{2 / 5}$, which translates into $\mu_{\mathrm{I}} \cong 1.48 \sigma_{0} \equiv \mu_{\mathrm{I}}^{\mathrm{c}}$. As shown in the figure, the $\mathrm{CP}$ comes across the line expressing the firstorder phase transition between the PIC and $\chi \mathrm{SB}$ phases. This means when $\mu_{\mathrm{I}}$ becomes large, the chiral critical point could fade out from the QCD phase diagram. The ratio of critical $\mu_{\mathrm{I}}$ to $\sigma_{0}$, the chiral condensate at the $\mathrm{CP}$, can be numerically evaluated as $\sim 1.48$, which is universal being independent of any GL parameters to this order.

\section{CONCLUSION}

We performed a systematic GL analysis on the effect of isospin asymmetry on the chiral crossover, the $\mathrm{CP}$ and its neighborhood. We first focused on how the crossover is affected by the isospin density. To this aim, we derived a general GL potential up to the quartic order in two chiral four-vectors, $\phi$ and its parity partner $\varphi$. Making use of the quark loop approximation together with a perturbative expansion in $\mu_{\mathrm{I}}$, we have studied not only the nature of the phase transition to the PIC but also how it is affected by the effect of the $\mathrm{U}(1)_{\mathrm{A}}$ anomaly. We found the effect of the isospin-flip odd $\lambda$ term in the potential makes the phase transition to first order at large $\lambda$. Since $\lambda$ vanishes at $\mu=0$ and increases with $\mu$, this may explain why the transition to the PIC is observed to be first order at finite $\mu$ in several model analyses [6, 7]. The effect of flavor mixing due to the $\mathrm{U}(1)_{\mathrm{A}}$ anomaly was found to diminish the effect of the $\lambda$ term by locking two condensates, $\sigma_{u}$ and $\sigma_{d}$. We have derived three model-independent universal ratios $-\mu_{\mathrm{I}}^{\mathrm{c}} / \sigma_{\mathrm{pc}}$ at $\lambda=0$, $\lambda^{\mathrm{TCP}} / \sigma_{\mathrm{pc}}$ and $\mu_{\mathrm{I}}^{\mathrm{TCP}} / \sigma_{\mathrm{pc}}$ at the $\mathrm{TCP}-$, which are independent of any GL parameters to the fourth order.

We then extended the analysis up to the sixth order of GL expansion, so as to study the isospin effect on 
the CP. Restricting the analysis to the case with strong $\mathrm{U}(1)_{\mathrm{A}}$ symmetry breaking, we studied how the CP and its neighborhood are affected by the incorporation of isospin density. We found that it has remarkable effects; it not only causes a shift of the location of the $\mathrm{CP}$, but also brings about the development of a sizable region for the homogeneous pion condensate. This leads to the appearance of new tricritical and triple points. Moreover we showed that the $\mathrm{CP}$ disappears once the isospin chemical potential is increased above a critical value. We derived the critical value $\mu_{\mathrm{I}}^{\mathrm{c}}$ and a universal relation relating it with the size of chiral condensate at the $\mathrm{CP}, \sigma_{0}$.

There are several directions into which the current work can be extended. First, we need to take into account the possibility of inhomogeneous phases since they are known to play an important role near the TCP/CP [16, 23 28]. This is actually now under investigation [14]. Second, the extension to three flavors would be inter- esting. This would require the incorporation of a kaon condensate and a diquark condensate of the color-flavor locked type. In particular, it is known that the interplay between the chiral and diquark condensates via the axial anomaly leads to a rich variety of phases and an appearance of new multicritical points [29, 30]. Third, the effect of vector interaction should be taken into account [31, 32]. This is especially needed when we look at dynamical aspects of the critical behavior. In fact the dynamic universality class of the chiral CP is known to be the same as the liquid-gas CP [33, 34]. Lastly, it is strongly desirable to seek the stiff experimental signatures of critical points observed here, such as those discussed for CP [35].

The author thanks K. Suzuki for several useful comments. A part of numerical calculations was carried out on SR16000 at YITP in Kyoto University.
[1] M. Asakawa and K. Yazaki, Nucl. Phys. A 504, 668 (1989).

[2] K. Fukushima and T. Hatsuda, Rept. Prog. Phys. 74, 014001 (2011); R. Casalbuoni, Proc. Sci., CPOD2006, (2006) 001.

[3] See, for example, K. Iida, T. Matsuura, M. Tachibana, and T. Hatsuda, Phys. Rev. Lett. 93, 132001 (2004); H. Abuki, M. Kitazawa, and T. Kunihiro, Phys. Lett. B 615, 102 (2005); H. Abuki and T. Kunihiro, Nucl. Phys. A 768, 118 (2006).

[4] D. T. Son and M. A. Stephanov, Phys. Rev. Lett. 86, 592 (2001).

[5] L. -y. He, M. Jin and P. -f. Zhuang, Phys. Rev. D 71, 116001 (2005); for a relativistic BCS-BEC crossover, see Y. Nishida and H. Abuki, Phys. Rev. D 72, 096004 (2005); H. Abuki, Nucl. Phys. A 791, 117 (2007).

[6] B. Klein, D. Toublan, and J. J. M. Verbaarschot, Phys. Rev. D 68, 014009 (2003); D. Toublan and J. B. Kogut, Phys. Lett. B 564, 212 (2003).

[7] A. Barducci, R. Casalbuoni, G. Pettini, and L. Ravagli, Phys. Rev. D 69, 096004 (2004).

[8] Y. Nishida, Phys. Rev. D 69, 094501 (2004).

[9] L. -y. He, M. Jin, and P. -f. Zhuang, Mod. Phys. Lett. A 22, 637 (2007).

[10] L. He, M. Jin, and P. Zhuang, Phys. Rev. D 74, 036005 (2006).

[11] J. O. Andersen and L. Kyllingstad, J. Phys. G 37, 015003 (2010).

[12] H. Abuki, R. Anglani, R. Gatto, M. Pellicoro and M. Ruggieri, Phys. Rev. D 79, 034032 (2009).

[13] H. Abuki, T. Brauner, and H. J. Warringa, Eur. Phys. J. C 64, 123 (2009).

[14] H. Abuki, in preparation.

[15] Y. Iwata, H. Abuki, and K. Suzuki, arXiv:1206.2870, AIP Conf. Proc. 1492, 293 (2012).

[16] D. Nickel, Phys. Rev. Lett. 103, 072301 (2009).

[17] M. Frank, M. Buballa, and M. Oertel, Phys. Lett. B 562, 221 (2003).

[18] T. Schfer, D. T. Son, M. A. Stephanov, D. Toublan, and
J. J. M. Verbaarschot, Phys. Lett. B 522, 67 (2001).

[19] V. A. Miransky and I. A. Shovkovy, Phys. Rev. Lett. 88, 111601 (2002).

[20] B. Friman, Acta Phys. Polon. Supp. 5, 707 (2012).

[21] A. Ohnishi, H. Ueda, T. Z. Nakano, M. Ruggieri, and K. Sumiyoshi, Phys. Lett. B 704, 284 (2011).

[22] H. Ueda, T. Z. Nakano, A. Ohnishi, M. Ruggieri, and K. Sumiyoshi, arXiv:1304.4331.

[23] E. Nakano and T. Tatsumi, Phys. Rev. D 71, 114006 (2005).

[24] D. Nickel, Phys. Rev. D 80, 074025 (2009).

[25] S. Carignano, D. Nickel, and M. Buballa, Phys. Rev. D 82, 054009 (2010).

[26] H. Abuki, D. Ishibashi, and K. Suzuki, Phys. Rev. D 85, 074002 (2012); H. Abuki and K. Suzuki, AIP Conf. Proc. 1492, 288 (2012).

[27] S. Carignano and M. Buballa, Phys. Rev. D 86, 074018 (2012).

[28] K. Fukushima, Phys. Rev. D 86, 054002 (2012).

[29] T. Hatsuda, M. Tachibana, N. Yamamoto, and G. Baym, Phys. Rev. Lett. 97, 122001 (2006); H. Abuki, G. Baym, T. Hatsuda, and N. Yamamoto, Phys. Rev. D 81, 125010 (2010); P. D. Powell and G. Baym, Phys. Rev. D 85, 074003 (2012).

[30] For the stability of multicritical points suggested in Ref. 29], see H. Basler and M. Buballa, Phys. Rev. D 82, 094004 (2010); Z. Zhang and T. Kunihiro, Phys. Rev. D 83, 114003 (2011).

[31] M. Kitazawa, T. Koide, T. Kunihiro, and Y. Nemoto, Prog. Theor. Phys. 108, 929 (2002).

[32] Z. Zhang, K. Fukushima, and T. Kunihiro, Phys. Rev. D 79, 014004 (2009); Z. Zhang and T. Kunihiro, Phys. Rev. D 80, 014015 (2009).

[33] H. Fujii, Phys. Rev. D 67, 094018 (2003).

[34] D. T. Son and M. A. Stephanov, Phys. Rev. D 70, 056001 (2004).

[35] M. A. Stephanov, K. Rajagopal, and E. V. Shuryak, Phys. Rev. Lett. 81, 4816 (1998); Phys. Rev. D 60, 114028 (1999). 